Щ्)FRANÇAISE

$\supset$ DE

필 PEAGOGIE
Revue française de pédagogie

Recherches en éducation

$197 \mid 2016$

Les multiples facettes de la créativité dans

l'apprentissage

\title{
Outils didactiques et changement pédagogique : analyse longitudinale de l'appropriation de l'outil Lirécrire par des enseignants du secondaire
}

Didactic tools and educational change: longitudinal analysis of secondary school teachers' appropriation of the Lirécrire Tool

Jessica Penneman, Séverine De Croix, Sébastien Dellisse, Jean-Louis Dufays, Xavier Dumay, Vincent Dupriez, Benoît Galand et Marielle Wyns

\section{OpenEdition}

Journals

Édition électronique

URL : https://journals.openedition.org/rfp/5165

DOI : $10.4000 /$ rfp.5165

ISSN : 2105-2913

Éditeur

ENS Éditions

Édition imprimée

Date de publication : 31 décembre 2016

Pagination : 79-98

ISSN : 0556-7807

\section{Référence électronique}

Jessica Penneman, Séverine De Croix, Sébastien Dellisse, Jean-Louis Dufays, Xavier Dumay, Vincent

Dupriez, Benoît Galand et Marielle Wyns, « Outils didactiques et changement pédagogique : analyse longitudinale de l'appropriation de l'outil Lirécrire par des enseignants du secondaire », Revue française de pédagogie [En ligne], 197 | 2016, mis en ligne le 31 décembre 2019, consulté le 07 janvier 2022. URL : http://journals.openedition.org/rfp/5165 ; DOI : https://doi.org/10.4000/rfp.5165 


\title{
Outils didactiques et changement pédagogique : analyse longitudinale de l'appropriation de l'outil Lirécrire par des enseignants du secondaire
}

\author{
Jessica Penneman \\ Séverine De Croix \\ Sébastien Dellisse \\ Jean-Louis Dufays \\ Xavier Dumay \\ Vincent Dupriez \\ Benoît Galand \\ Marielle Wyns
}

Cet article porte sur le processus d'appropriation d'outils didactiques proposés à des enseignants. Après avoir exposé notre modèle théorique de l'appropriation d'un outil didactique et des facteurs susceptibles de l'influencer, nous présenterons les grandes lignes de la recherche Lirécrire dans laquelle s'inscrit cette contribution, ainsi que l'outil didactique qui a été proposé aux professeurs de français ayant collaboré à ce projet. En nous basant notamment sur l'analyse de focus-groupes, de séances d'accompagnement et d'entretiens semi-directifs menés auprès de 35 enseignants, nous montrons les effets induits par le recours à cet outil sur les conceptions de l'enseignement de la lecture et les pratiques pédagogiques de ces enseignants. Les résultats mettent en lumière différents niveaux d'appropriation de l'outil Lirécrire pour apprendre par les enseignants. II apparaît également que certains principes didactiques ont reçu un accueil plus favorable que les autres. II s'agit en particulier du recours à des textes informatifs et de l'enseignement explicite des stratégies de lecture.

Mots-clés (TESE) : pratique pédagogique, innovation pédagogique, développement des capacités professionnelles 


\section{Introduction}

En France, en Belgique et dans de nombreux autres pays, les résultats actuels des élèves aux épreuves institutionnelles portant sur la compréhension et la production de textes sont jugés globalement insuffisants. Plusieurs recherches (Bautier \& Goigoux, 2004; Cèbe \& Goigoux, 1999) suggèrent qu'une partie de l'explication tient aux pratiques dominantes d'enseignement de la lecture: peu de démarches centrées sur la lecture pour apprendre, recours à des textes fort courts, non intégraux, presque exclusivement narratifs, tâches très découpées ou très mécaniques (réponses brèves, textes lacunaires, choix multiples...). D'autres recherches (Crinon \& Marin, 2008; Falardeau \& Gagné, 2012; Nonnon, 2012; Van Grunderbeeck, Théorêt, Chouinard et al., 2004) soutiennent qu'il est possible d'aider les élèves en difficulté de lecture en agissant sur ces démarches : il s'agirait ainsi de clarifier la nature et le statut des documents utilisés dans la sphère scolaire, de varier les sujets des textes, d'enseigner explicitement des stratégies de compréhension, d'accompagner la lecture de tâches d'écriture, etc. Dans ce contexte, tant les recherches francophones (Bélanger, Bowen, Cartier et al., 2012; Bonnéry, 2015; Cartier, Contant \& Janosz, 2012) que les travaux issus du monde anglo-saxon (Berman, 1980; Jacobs \& Ippolito, 2015; Pao \& Williams, 2015; Rowan \& Miller, 2007) soulignent le rôle que peuvent jouer les outils didactiques en tant que supports au changement de pratiques pédagogiques. Dans de nombreux pays, des initiatives diverses existent d'ailleurs pour proposer aux enseignants des ressources didactiques (manuels, supports de travail pour la classe, ressources numériques...) dont les promoteurs espèrent qu'elles puissent canaliser le travail enseignant dans la direction souhaitée.

On sait toutefois que proposer aux enseignants des outils et des ressources didactiques de qualité présage peu de l'usage qui en sera fait et des bénéfices potentiels pour les apprentissages des élèves (Chartier, 2000; Dupriez, 2015). Les recherches nord-américaines ont abondamment traité cette question, en partant souvent du principe que les bénéfices les plus grands seront obtenus si les enseignants respectent fidèlement les prescriptions communiquées par les concepteurs des outils proposés. Néanmoins, plusieurs travaux relatifs au processus d'implémentation de réformes pédagogiques basées sur des outils montrent les limites de l'idée selon laquelle l'implémentation optimale repose enseignants et sur le respect du volume d'activités suggéré (Durlak \& DuPre, 2008; Dusenbury, Brannigan, Falco et al., 2003; Rowan \& Miller, 2007). En effet, ces outils didactiques ne produisent pas systématiquement les effets attendus sur les apprentissages des élèves, en raison de la double médiation qui sépare les outils et les visées : d'une part, l'appropriation des outils par l'enseignant implique des adaptations liées au contexte et, d'autre part, l'usage de ces outils par les élèves dépend de multiples facteurs motivationnels et organisationnels (Dupriez, 2015).

La recherche présentée dans cet article repose plutôt sur l'hypothèse que les ressources didactiques proposées aux enseignants font l'objet d'un travail d'appropriation progressive par ceux-ci. C'est la manière dont les enseignants se sont emparés de l'outil et la façon dont ils ont potentiellement transformé les démarches proposées que nous étudions ici. Dans une première section du texte, nous veillons à rendre explicite notre conception d'un tel processus et des facteurs qui peuvent l'influencer. Nous présentons ensuite brièvement la recherche intitulée Lirécrire ${ }^{1}$ et la manière dont nous avons étudié le processus d'appropriation de l'outil promu par cette recherche. Les résultats sont organisés autour de deux questions : $1^{\circ}$ comment les enseignants s'approprient-ils un outil didactique particulier? et $2^{\circ}$ quels sont les facteurs qui influencent cette appropriation?

\section{Cadre théorique : vers un modèle théorique du processus d'appropriation}

\section{L'appropriation d'outils didactiques définie dans la littérature}

L'appropriation est appréhendée ici en tant que processus complexe "d'intégration, partielle ou totale, d'un objet ou d'un dispositif à la culture propre de l'acteur » (Theureau, 2011, p. 11). Ce processus s'effectue par phases, par étapes progressives durant lesquelles l'enseignant va simultanément interpréter et transformer l'outil mis en œuvre, mais également modifier ses

1 Le projet Lirécrire, subventionné par la Fondation Louvain (UCL), est une recherche développée en Belgique francophone par Séverine De Croix, Jean-Louis Dufays, Xavier Dumay, Vincent Dupriez et Benoît Galand (promoteurs), avec la participation de Sébastien Dellisse, Jessica Penneman et Marielle Wyns (chercheurs). 


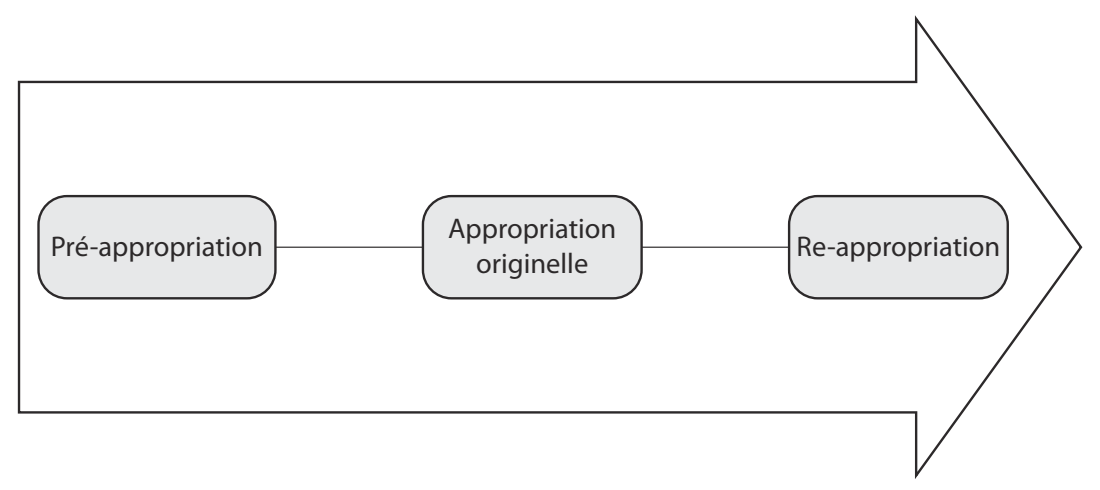

perceptions initiales et ajuster ses pratiques d'enseignement (Bélanger, Bowen, Cartier et al., 2012; Dupriez, 2015; Duthoit \& Metz, 2012). L'appropriation n'est donc pas le résultat d'une application mécanique de propositions prescrites.

Les recherches qui se sont intéressées à l'appropriation d'outils ou de nouvelles pratiques (Bélanger, Bowen, Cartier et al., 2012; de Vaujany, 2006; Duthoit \& Metz, 2012) considèrent que ce processus s'inscrit dans une temporalité plus ou moins longue. De Vaujany (2006) propose de représenter ce processus d'appropriation en trois phases schématisées dans la figure 1.

Pour de Vaujany (2006), la pré-appropriation débute avant même l'utilisation ou la mise en œuvre d'un nouvel outil. Elle correspond à la mise en projet, à la première interprétation, et elle résulte des discussions et des évocations relatives à l'outil à mettre en œuvre (à l'occasion, par exemple, de formations, de présentations...). Elle se traduit par la prise de conscience de I'intérêt de changer ses pratiques et aboutit à l'acceptation d'entrer dans le projet, dans le dispositif offert par les promoteurs d'un outil (considérés alors comme un «soutien externe») et de mettre en œuvre les démarches proposées.

Suit alors l'étape d'appropriation originelle. Cette phase, caractérisée par l'émergence de tensions et de conflits entre les perceptions initiales des acteurs concernés par le changement et les caractéristiques mêmes de l'outil, renvoie à un changement de pratiques : le professionnel va interpréter et transformer I'outil et ajuster ses pratiques en fonction du contexte environnemental et individuel dans lequel l'outil est mis en œuvre.

Enfin, la phase de ré-appropriation correspond à l'évolution de l'outil. Les nouvelles pratiques se combinent aux pratiques antérieures et sont réinvesties régulièrement dans de nouvelles situations. À ce moment du processus d'appropriation, le soutien externe (formation ou accompagnement apporté par les concepteurs de l'outil ou les formateurs) et les outils proposés ne sont plus indispensables. Dans cette phase, l'appropriation d'outils ou de nouvelles pratiques continue bien au-delà des premiers signes de changement de pratiques.

\section{Les facteurs influençant l'appropriation d'outils didactiques}

Les recherches centrées sur l'analyse de l'évolution des programmes d'intervention ont mis en évidence divers facteurs associés à leur mise en œuvre (Desimone, 2002; Durlak \& DuPre, 2008; Dusenbury, Brannigan, Falco et al., 2003; Ringwalt, Ennett, Johnson et al., 2003). Sans prétendre à l'exhaustivité, cinq catégories de facteurs spécifiques à l'appropriation de nouvelles pratiques ont retenu notre attention: $1^{\circ}$ les facteurs liés à l'enseignant, $2^{\circ}$ les facteurs liés à l'outil, $3^{\circ}$ les facteurs liés à la classe, $4^{\circ}$ les facteurs liés à l'environnement organisationnel, et $5^{\circ}$ les facteurs liés au soutien externe.

L'enseignant est au cœur du développement de pratiques pédagogiques puisque c'est lui qui va recourir aux outils qui lui sont proposés. La valeur qu'il accorde à l'outil (notamment en termes de bénéfices pour les apprentissages des élèves), ses compétences à le mettre en œuvre (en termes de connaissances et de sentiment d'efficacité personnelle), ses représentations du métier (en termes de croyances et de conceptions de l'enseignement) et ses pratiques antérieures ont un impact sur I'appropriation d'outils didactiques (Durlak \& DuPre, 2008; Goigoux, 2007; Ringwalt, Ennett, Johnson et al., 2003; Spillane, Reiser \& Reimer, 2002). Des pratiques, des valeurs, des conceptions et des représentations de 
l'enseignant trop éloignées d'un outil promu impliqueront une réorganisation de ces dernières trop importante pour espérer une appropriation complète et durable des propositions didactiques et pédagogiques (Cèbe \& Goigoux, 2007).

Les caractéristiques liées à l'outil ont aussi une influence (Cèbe \& Goigoux, 2007; März, 2014). En effet, les recherches antérieures (Cèbe \& Goigoux, 2007; Durlak \& DuPre, 2008) montrent l'importance de proposer des outils qui rencontrent des critères d'intelligibilité (s'inscrire dans le genre professionnel des enseignants), d'efficacité (valoir aux professeurs un minimum d'avantages) et de proximité (ne pas être trop éloigné des pratiques des enseignants). Pour être approprié, un outil gagne à pouvoir être adapté au contexte, à répondre aux besoins des élèves et des enseignants et à être cohérent avec les prescrits institutionnels. L'outil mis entre les mains des enseignants revêt également une fonction de prescription. Dans ce cadre-là, tout en permettant des adaptations liées au contexte, les objectifs à atteindre ainsi que les stratégies à mettre en place pour y parvenir ont intérêt à être clairement définis (Durlak \& DuPre, 2008; Dusenbury, Brannigan, Falco et al., 2003; Fullan, 2015; Goigoux, 2007).

Les caractéristiques des élèves (leur nombre, leur niveau de compétences initiales, leur motivation...) et du climat de classe ont également une influence sur le devenir de propositions pédagogiques et didactiques. Elles affectent l'appropriation de l'outil (notamment au niveau des adaptations qui y seront apportées) et l'engagement de l'enseignant dans le projet (Janosz \& Deniger, 2001 ; Janosz, Georges \& Parent, 1998).

Enfin, des caractéristiques liées à l'environnement organisationnel jouent aussi un rôle non négligeable dans la réussite ou l'échec de changements de pratiques (Durlak \& DuPre, 2008). II s'agit du contexte organisationnel général (relations avec les collègues, rapport à l'innovation de l'enseignant et de l'équipe pédagogique, structure organisationnelle de l'établissement, valeurs partagées par le corps enseignant...), mais également du degré de confiance entre les membres de l'équipe éducative et du soutien de la direction. La collaboration entre les enseignants semble également être un facteur déterminant dans le développement et l'appropriation de nouvelles pratiques, en particulier lorsque celle-ci rend les pratiques de classe visibles, c'est-à-dire lorsque le travail collaboratif est centré sur la production d'activités de classe et/ou l'analyse de traces de l'activité enseignante (Horn \& Little, 2010).
Dans un processus de changement de pratiques et de développement professionnel, de plus en plus de recherches s'accordent sur la nécessité de proposer aux équipes éducatives un soutien externe dans la mise en œuvre et l'appropriation d'outils (Charlier \& Biémar, 2012; Corriveau, Letor \& Bagnoud, 2010; Dupriez, 2015; Letor, 2009; Rowan \& Miller, 2007...). Celui-ci peut prendre la forme d'une formation et/ou d'un accompagnement spécifique (Bélanger, Bowen, Cartier et al., 2012 ; Dupriez, 2015 ; Letor, 2009). Les formations les plus efficaces semblent être conçues pour prendre en considération les attentes des enseignants, leur motivation et leur sentiment d'efficacité personnelle face à l'intervention promue (Durlak \& DuPre, 2008; Dusenbury, Brannigan, Falco et al., 2003). Les dispositifs d'accompagnement permettent un travail dans la durée, centré sur le partage de pratiques et sur la compréhension des expériences positives et des difficultés rencontrées lors de l'usage de l'outil proposé (Horn \& Little, 2010).

\section{Contexte de la recherche Lirécrire}

L'analyse présentée dans cet article s'appuie sur les données d'une recherche longitudinale en milieu naturel, visant à développer des pratiques d'enseignement susceptibles de favoriser la compréhension et la production des textes informatifs des élèves du début du secondaire (entre 12 et 13 ans). Plus précisément, le projet Lirécrire a pour objectifs de soutenir, durant deux années scolaires consécutives (2014-2016), la mise en œuvre d'un outil didactique, d'analyser le processus d'appropriation de cet instrument par les enseignants et d'évaluer la capacité de ce dispositif à améliorer les compétences des élèves. C'est le deuxième objectif, I'analyse du processus d'appropriation, qui est traité dans cet article.

Dansle cadre de cette recherche, deux chercheusesdidacticiennes ont conçu un outil didactique en trois volumes (guide méthodologique détaillé pour l'enseignant, activités et documents pour les élèves). Destiné aux élèves de première secondaire ( $7^{\mathrm{e}}$ année de scolarité), Lirécrire pour apprendre (De Croix \& Penneman, 2014) a été construit en relation avec les instructions officielles actuelles en Belgique francophone et vise à développer les compétences en lecture des textes informatifs requises pour réussir à l'école. Ce prototype, représentant une cinquantaine d'heures de cours, autorisant des choix personnels de l'enseignant, se structure en cinq modules et s'organise autour de 


\begin{tabular}{l|l}
\hline \multicolumn{2}{l}{ Structure de l'outil Lirécrire pour apprendre } \\
\hline Module 1 & Se familiariser avec les usages des écrits à visée informative, explicative, justificative \\
\hline Module 2 & Sélectionner des informations dans un ou plusieurs textes simultanément et les lier entre elles \\
\hline Module 3 & Rappeler, reformuler, résumer un texte et échanger à son propos \\
\hline Module 4 & Organiser, linéariser l'information issue d'une double page de manuel \\
\hline Module 5 & Répondre à des questions sur un texte et justifier ses réponses \\
\hline \multicolumn{2}{l}{ Principes didactiques de l'outil Lirécrire pour apprendre }
\end{tabular}

Favoriser l'engagement cognitif et l'implication des élèves en recourant à une variété de documents à visée informative, le plus souvent intégraux, composites et diversifiés.

Expliciter les stratégies de lecture (lecture de survol, lecture repérage, lecture intégrale linéaire...), les processus de lecture (sélection des informations essentielles, liaison des informations, formulation d'hypothèses, mobilisation de connaissances, inférences...) et les opérations de production de textes (planification, textualisation, révision).

Faire acquérir de bonnes habitudes de lecture en proposant un entraînement spiralaire (répétition des démarches et travail sur les stratégies de lecture à plusieurs reprises) et progressif (de consignes très cadrées à des tâches de plus en plus complexes et globales).

Favoriser les échanges et les interactions ouvertes et alterner les modes de travail (en sous-groupes, collectif ou individuel).

Faire prendre conscience aux élèves de leur fonctionnement en lecture-écriture et des régulations possibles en les amenant à raisonner sur leur propre activité au moyen de tâches centrées sur la métacognition et sur l'autorégulation.

Favoriser le transfert des compétences en confrontant l'élève à des situations authentiques, scolaires ou extrascolaires, de lecture-écriture de textes informatifs.

six principes didactiques largement reconnus comme efficaces (sur l'apprentissage des élèves) dans la littérature scientifique ${ }^{2}$. La structuration de l'outil et les principes didactiques sont résumés dans le tableau $1^{3}$.

Au départ, 19 établissements se sont portés volontaires pour participer à cette recherche, acceptant, d'une part, d'utiliser durant deux années consécutives cet outil dans le cadre du cours de français destiné aux élèves de $1^{\text {re }}$ année du secondaire et, d'autre part, d'entrer dans un contexte particulier de formation (et, pour certains enseignants, dans un dispositif d'accompagnement) et de recherche (répartition aléatoire des écoles dans les groupes, contraintes temporelles et

2 Lirécrire pour apprendre repose sur plusieurs principes inspirés des dispositifs didactiques centrés sur les textes narratifs conçus par S. Cèbe et R. Goigoux (2009) mais également sur les recherches de D. Bucheton (2014), É. Falardeau et J.-C. Gagné (2012).

3 Le lecteur trouvera une description plus précise de l'outil Lirécrire pour apprendre, de ses principes et de ses fondements théoriques dans l'article intitulé "Concevoir un outil didactique pour enseigner la lecture des textes informatifs au début du secondaire» publié dans le $35^{\mathrm{e}}$ numéro de la revue Diptyque (De Croix, Penneman \& Wyns, 2017). organisationnelles liées aux prises de mesure $\left.{ }^{4} . ..\right)$. Dans chacune de ces écoles, les professeurs de français concernés ont pu exprimer leur souhait de participer à la recherche à travers un vote secret. La recherche ne débutait que si elle récoltait au moins $60 \%$ de réponses positives. Les établissements participants ont ensuite été aléatoirement distribués dans trois groupes : un groupe «Témoin » qui a bénéficié de l'outil un an après

$4 \quad$ Les prises de mesure ont été très nombreuses tout au long de la recherche et ont constitué, pour les enseignants, des contraintes fortes. Pour mesurer les effets de l'intervention sur les élèves et les enseignants et identifier les variables qui influencent ces effets, plusieurs outils de recueil de données ont été construits : une épreuve de compétences en lecture-écriture qui évalue les performances des élèves avant et après l'intervention (pré- et post-tests), des questionnaires portant sur le rapport à la lecture-écriture des élèves et des enseignants, sur les pratiques ordinaires d'enseignement, sur la perception de l'école et l'environnement organisationnel et sur le degré d'implémentation de l'outil didactique. Parallèlement à ces données, des focus-groupes, des séances d'accompagnement, des questionnaires de fin de formation et d'accompagnement, des entretiens individuels et des traces de l'activité des élèves et des enseignants recueillies en cours de recherche apportent des informations qualitatives sur la mise en œuvre et l'appropriation de l'outil par les enseignants. 
les autres (et qui ne donne pas lieu à une analyse dans cet article), un groupe "Formation» et un groupe «Accompagnement ». Avant d'expérimenter les différentes activités de l'outil, les membres du groupe «Formation » ont suivi une formation de dix heures, réparties sur deux journées (à un mois d'intervalle). Au regard des travaux de Dusenbury, Brannigan, Falco et alii (2003), de Durlak et DuPre (2008) et de Biesta (2007), la formation proposée était centrée sur la découverte de l'outil, sur l'utilisation et la manipulation du matériel didactique, sur l'expérimentation de quelques activités et sur les fondements théoriques qui ont guidé sa construction. Les enseignants du groupe «Accompagnement » ont bénéficié de la même formation (mais séparés du groupe «Formation » pour éviter le biais de contamination des groupes), mais ont en outre été accompagnés collectivement par les chercheurs durant la première année de travail. Cet accompagnement de neuf heures (réparties en trois séances) a été organisé entre janvier et mai 2015 au sein de chaque établissement et visait, notamment, à guider les enseignants dans la mise en œuvre de l'outil et à renforcer les effets attendus par le dispositif (en termes d'effets sur les apprentissages des élèves et sur l'appropriation de l'outil par les enseignants) (Rowan \& Miller, 2007). Construit en relation avec le modèle d'accompagnement défini par Charlier et Biémar (2012), chaque séance d'accompagnement se structure en trois temps et vise les objectifs suivants:

1. temps d'échange: prise de contact, rappel du contrat, explicitation des besoins des enseignants, état des lieux de ce qui a été fait, etc.;

2. temps rétrospectif : à partir des traces des élèves, travail sur un objet en particulier (l'évaluation, le questionnaire de lecture, les difficultés de lecture-écriture des élèves...), retour sur les difficultés rencontrées par les élèves et par les enseignants, partage de pratiques et d'expériences positives;

3. temps prospectif : planification des activités, compréhension des implications didactiques liées aux fondements théoriques de l'outil, clarification des modalités de mise en œuvre de I'outil et des objectifs à atteindre, etc.

\section{Le modèle d'analyse de l'appropriation dans le cas de Lirécrire}

Inspirée de la proposition de de Vaujany (2006), la processus d'appropriation de l'outil et des facteurs susceptibles de l'influencer.

L'entrée dans le projet correspond à l'étape de «préappropriation» définie par de Vaujany (2006). Dans le cas de Lirécrire, cette entrée dans le projet correspond, d'une part, à une phase d'information des directions d'établissements scolaires et de leurs professeurs de français du début du secondaire à propos du dispositif de recherche et de la proposition didactique sousjacente et, d'autre part, à un moment décisionnel d'entrer dans le projet pour deux années consécutives caractérisé par le vote des enseignants. À la suite de la formation des enseignants à l'utilisation de l'outil Lirécrire pour apprendre, l'implémentation représente, pour les enseignants, la première étape du processus d'appropriation. Cette étape, induite par le dispositif de travail et par le soutien externe dont les enseignants ont bénéficié (uniquement la formation ou la formation et l'accompagnement), les pousse à utiliser l'outil en s'appuyant sur nos consignes et nos recommandations. Il s'agit pour eux de mettre en œuvre «fidèlement » les propositions didactiques et d'agir conformément à des consignes externes présentées lors de la formation et explicitées plus largement dans le guide méthodologique de l'enseignant ou lors de l'accompagnement (pour les enseignants du groupe «Accompagnement »). Cependant, la rencontre entre un enseignant singulier, une classe spécifique et l'outil proposé conduit, si l'enseignant s'engage à mettre en œuvre cet outil, à une phase d'intégration individuelle et personnelle de l'outil didactique. Cette intégration peut mener à des transformations et des modifications plus ou moins importantes de l'outil. Ces transformations, si elles sont cohérentes avec les principes didactiques de l'outil, amènent elles-mêmes à une appropriation de l'outil qui peut être observée tant sur le plan cognitif que comportemental. L'appropriation cognitive renvoie à la valeur accordée aux principes didactiques, pédagogiques et méthodologiques de l'outil à mettre en œuvre. Autrement dit, cette appropriation correspond à ce que les enseignants pensent de l'outil et manifestent à travers leurs discours (l'évocation de l'outil). L'appropriation comportementale renvoie aux comportements des enseignants, à ce qu'ils font de l'outil, et elle passe par des indicateurs relatifs à sa mise en œuvre ou à l'adoption de démarches conformes aux principes didactiques sous-jacents. Enfin, comme dans la phase de «réappropriation » définie par de Vaujany (2006), le transfert constitue une étape au-delà de l'appropriation, inscrite dans le développement professionnel de 


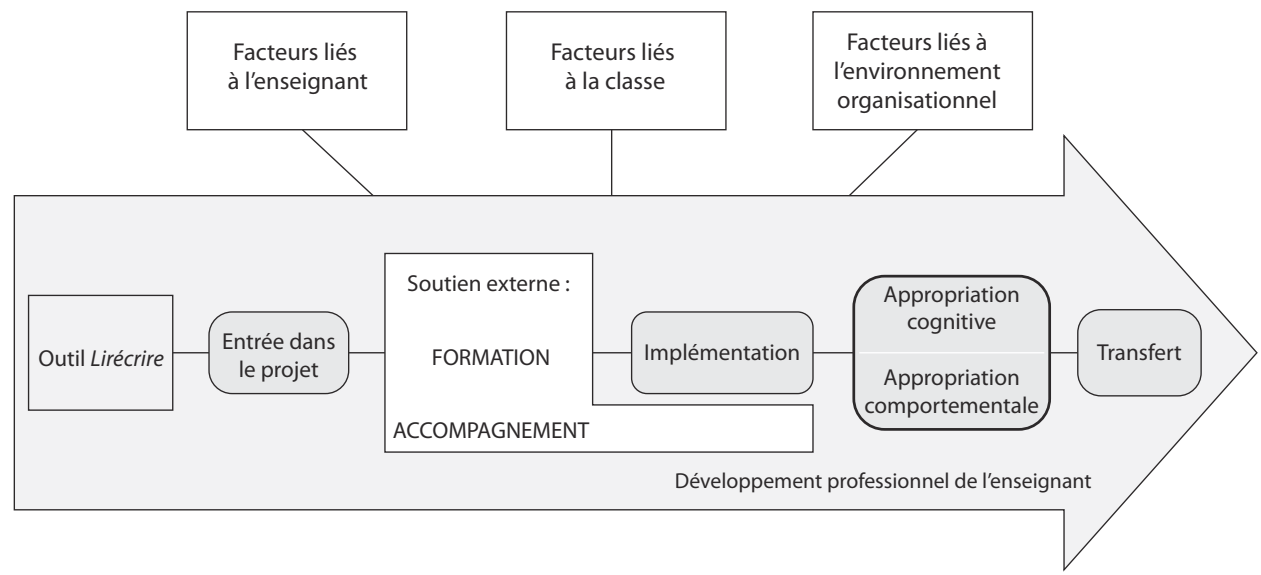

Tableau 2. Répartition de l'échantillon

\begin{tabular}{l|l}
\hline Groupe «Formation» & Groupe «Accompagnement \\
\hline 6 écoles & 5 écoles \\
\hline 21 enseignants & 14 enseignants \\
\hline
\end{tabular}

l'enseignant. II se réalise lorsque l'enseignant a intégré certains principes de l'outil qu'il traduit dans d'autres pratiques (dans d'autres cours de français, années d'enseignement ou disciplines). Enfin, comme nous l'avons vu, ce processus complexe est influencé par une diversité de facteurs liés à l'outil, au type de soutien externe proposé, mais également aux caractéristiques de l'enseignant, de la classe et de l'environnement organisationnel dans lequel l'outil est mis en œuvre.

\section{Objectifs et méthodologie}

\section{Objectifs et questions de recherche}

Notre recherche a un double objectif : analyser le processus d'appropriation d'un outil didactique et identifier les facteurs impliqués dans ce processus. Plus précisément, elle vise à répondre aux questions suivantes : $1^{\circ}$ comment les enseignants s'approprient-ils un outil didactique particulier ? et $2^{\circ}$ quels sont les facteurs qui influencent cette appropriation?

\section{Échantillon}

Pour répondre à ces questions, nous nous appuyons sur les données récoltées auprès des 49 enseignants volontaires aléatoirement affectés au groupe «Formation » ou au groupe «Accompagnement » et qui ont expérimenté l'outil Lirécrire pour apprendre. Parmi ces 49 enseignants (24 enseignants dans le groupe «Formation » et 25 dans le groupe «Accompagnement»), 7 ont quitté leur établissement, ont cessé d'enseigner (départ à la retraite) ou ont changé d'attributions en cours de recherche. Ils ont dès lors été soustraits de l'échantillon. 7 autres enseignants, qui ont été absents lors de plusieurs collectes de données, ont également dû être retirés de l'échantillon. Notre étude se base dès lors sur un échantillon composé de 35 enseignants pour lesquels nous disposons de très nombreuses données collectées durant deux années scolaires (voir tableau 2). Par souci de confidentialité, nous avons attribué un nom d'emprunt à chaque enseignant de l'échantillon.

\section{Collecte de données et méthode d'analyse}

L'essentiel du matériau analysé concerne les verbalisations des enseignants recueillies à l'occasion de diverses prises d'informations, auxquelles tous les enseignants s'étaient engagés à participer : des focus-groupes menés au sein de chacun des établissements (années 1 et 2$)^{5}$, des questionnaires de fin de formation et

5 Dans cette recherche, les focus-groupes ont rassemblé les enseignants d'une même école au terme de chacune des deux 


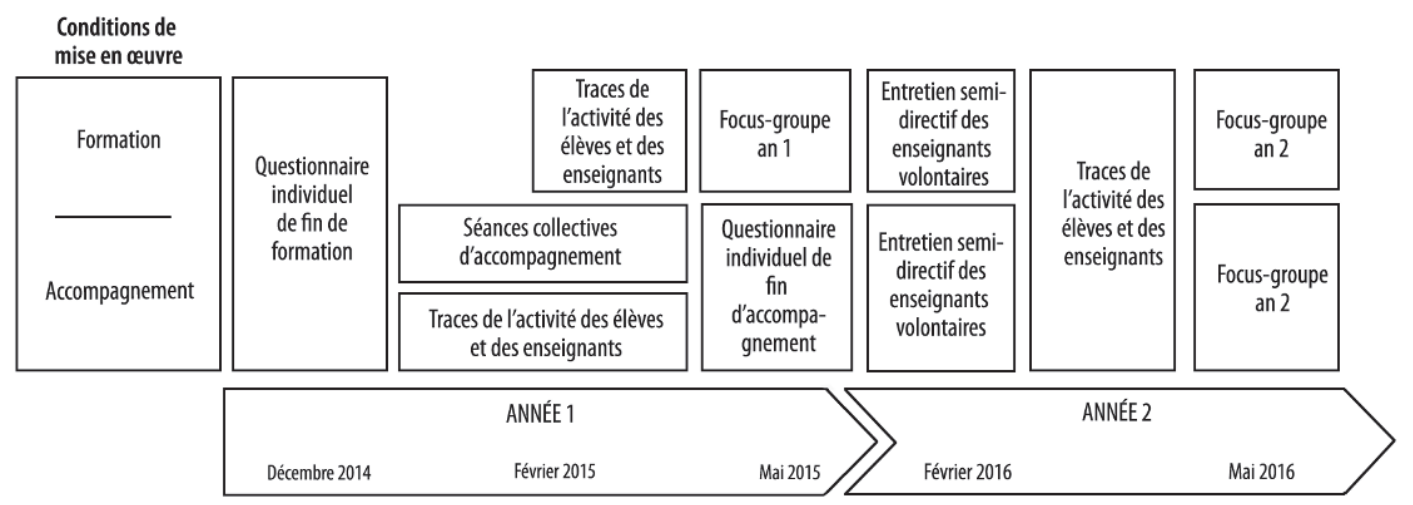

d'accompagnement (année 1) et des séances d'accompagnement (année 1). En outre, des traces de l'activité des élèves (activités complétées, affiches collectives...) et des enseignants (annotations du guide méthodologique, évaluations...) ont pu être rassemblées et analysées, et des entretiens semi-directifs de 16 enseignants volontaires (7 entretiens dans le groupe «Formation», 9 entretiens dans le groupe "Accompagnement») ont pu être réalisés la deuxième année, complémentairement aux données déjà collectées (voir figure 3).

Ces données collectées aux différentes étapes de la recherche avaient pour objectif de rendre compte de l'usage de l'outil didactique par les enseignants, des difficultés rencontrées et des réactions face à ces difficultés. Les questions posées aux enseignants visaient également à documenter leur perception du rôle de chacun des facteurs énoncés précédemment (voir figure 2) dans leur appropriation de l'outil.

Comme le synthétise la figure 4, les données recueillies lors de la première année de mise en œuvre de l'outil ont été soumises à une analyse catégorielle préliminaire, dans le but de confronter le matériau empirique récolté avec les choix théoriques posés. Cette première analyse a permis d'élaborer deux grilles

années respectives d'expérimentation (en mai-juin) et ont permis, grâce aux questions posées par le chercheur (par exemple : quelles difficultés avez-vous rencontrées dans la mise en œuvre de l'outil et qu'avez fait pour les surmonter? Quels sont les paramètres qui ont facilité la mise en œuvre de l'outil? Comment avez-vous vécu la mise en œuvre de l'outil? Qu'avez-vous fait pour vous approprier I'outil ? Sur quels critères avez-vous modifié l'outil ?...), de récolter de l'information sur la mise en œuvre et l'appropriation de l'outil d'analyse articulées au cadre théorique et dotées d'indicateurs spécifiques. La première grille porte sur l'appropriation cognitive et comportementale des six principes didactiques de l'outil (voir annexe 1) et la seconde sur les facteurs associés au processus d'appropriation de l'outil (voir annexe 2). L'ensemble des matériaux collectés a par ailleurs été retranscrit, classé et analysé en fonction de cette double grille, et à l'aide du logiciel Nvivo11.

Cette analyse a été réalisée dans un premier temps pour chacun des enseignants et a permis ensuite de construire une analyse globale pour l'ensemble des enseignants. En considérant tous les enseignants, trois groupes sont apparus très clairement. Ainsi, comme nous le verrons dans la partie «Résultats», pour une minorité d'enseignants, nous observons des indices d'appropriation tant cognitive que comportementale des six principes de l'outil Lirécrire. Dans les résultats, ce sont les enseignants pour lesquels nous dirons qu'ils s'approprient globalement l'outil. Pour un autre groupe d'enseignants, nous avons extrêmement peu d'indices d'appropriation tant cognitive que comportementale des six principes. Dans les résultats, nous parlerons de ces enseignants en disant que ceux-là ne se sont pas approprié l'outil. Enfin, un troisième groupe apparaît dans une position intermédiaire : nous avons relevé auprès de certains enseignants des indices d'appropriation tant cognitive que comportementale, mais qui se limitent principalement à deux des six principes de l'outil Lirécrire. Cette catégorie regroupe les enseignants qui se sont approprié partiellement l'outil. 


\section{Résultats}

L'analyse du processus d'appropriation de l'outil par les enseignants fait apparaître des groupes assez contrastés. Les résultats présentés ci-dessous tiennent compte de ce contraste en distinguant, d'une part, les enseignants qui montrent très peu (ou ne montrent pas du tout) d'indices d'appropriation et, d'autre part, les enseignants qui font preuve d'une appropriation au moins partielle de l'outil et de ses principes. Nous revenons ensuite sur les facteurs impliqués dans ce processus d'appropriation.

\section{Du côté des enseignants qui ne s'approprient pas l'outil}

Les premières observations qui ressortent de l'analyse qualitative mettent en évidence que certains enseignants résistent à l'outil et au changement de pratiques qu'il est susceptible d'induire. Le refus de mettre en œuvre les activités proposées ou le maintien de pratiques antérieures témoignent de cette nonappropriation. Ainsi, on constate que, sur les 35 enseignants engagés dans le projet, 14 (dont 10 du groupe «Formation») ne se sont pas approprié l'outil.
Parmi eux, au terme de la première année, cinq enseignants du groupe «Formation» (dont une équipe pédagogique complète composée de quatre enseignants) ont fait le choix de ne pas poursuivre la recherche Lirécrire. Outre la non-réalisation des activités proposées et parfois une contestation des principes sous-jacents à ces activités, ces enseignants avancent diverses raisons qui les empêchent de s'approprier I'outil : le manque de collaboration dans l'équipe éducative et les contraintes liées à la recherche :

On n'a pas d'équipe. [...] Je n'ai plus envie non plus de me lier à vous, avec des rapports, un nombre d'heures à donner, des trucs à lire, des évaluations... Je n'ai plus envie de revivre une expérience négative. Ça m'angoisse. Se tromper une fois, je veux bien, mais deux fois, ça n'a pas de sens (focus-groupe an 1, Pierrot).

Trois autres enseignants manifestent simultanément une distance comportementale (la seconde année, ils ne mettent en œuvre que très peu d'activités) et cognitive (ils expriment leur désaccord avec plusieurs principes associés à l'outil tels que la mise en place d'activités de métacognition ou d'activités en sous-groupes).

Enfin, les six derniers enseignants de cette première catégorie ont mis en œuvre les activités de 
l'outil, ont respecté les engagements vis-à-vis de la recherche (passer par tous les modules, être présents lors des passations d'épreuves et de questionnaires, s'engager pour deux ans...) et ont apprécié certaines démarches proposées dans l'outil (notamment liées aux stratégies de lecture ou aux textes informatifs). Mais la distance importante entre leurs pratiques antérieures habituelles et les propositions didactiques de Lirécrire est demeurée un obstacle à une réelle intégration et appropriation de l'outil :

Mais je pense qu'ils [ses collègues] ont plus de facilités à rentrer dans un cours comme ça parce que ce sont des plus jeunes. Moi, je suis beaucoup plus classique, avec une façon de faire depuis des années. Et c'est pour ça que, parfois, j'ai été déconcertée. J'ai quelquefois paniqué aussi face à ce qu'on me demandait. C'était tellement différent de ce que je faisais avant que j'ai paniqué (focus-groupe an 2 , Gentiane).

Certaines pratiques ordinaires considérées par les enseignants comme éloignées des principes didactiques de l'outil ont des répercussions sur la façon de mener les activités. Ainsi, l'analyse des transformations apportées aux activités témoigne d'une tendance à rapprocher les propositions didactiques de l'outil des pratiques antérieures de ces enseignants. Ces transformations ne permettent pas de respecter les principes didactiques de l'outil. C'est le cas notamment du travail en sous-groupes, qui permet aux élèves en difficulté ou plus passifs d'activer ou d'utiliser les stratégies qu'ils n'utilisent généralement pas, de contrôler davantage, de façon collective, la tâche qui leur est soumise et par conséquent, d'améliorer leurs compétences en compréhension de textes (Langenberg, 2000; Snow, 2002). Pour des questions d'habitude ou de gestion de classe, certains enseignants les évitent et privilégient les activités individuelles:

Moi, les travaux de groupe, je suis désolée, mais c'est insupportable. Pour moi, c'est quelque chose d'horrible. Ils parlent tous en même temps. C'est insupportable. [...] Cette année, sincèrement, je l'ai très peu fait. L'activité avec le voisin et tout, non. C'est tout seul (focus-groupe an 2, Clarence).

Pour gagner du temps ou pour laisser davantage de place à la lecture des textes narratifs, les enseignants ont également fait le choix de sélectionner certaines activités sans tenir compte des objectifs à atteindre ou de la progression des apprentissages. C'est le cas pour les activités centrées sur la métacognition ou sur l'entraînement «spiralaire», généralement supprimées par
J'ai fait le Lirécrire en condensé pour pouvoir redonner plus de place au narratif (focus-groupe an 2, Yasmine).

C'est vrai que moi, par exemple, tout ce qui était "retour réflexif», etc., aussi pour une question de temps, j'ai tendance un peu à zapper (focus-groupe an 2, Laetitia).

D'autres modifications ont également pu être observées. Il en va ainsi de l'évaluation des apprentissages des élèves. Lirécrire ne propose pas d'outils d'évaluation. Certains enseignants ont alors soit utilisé certaines activités de l'outil pour évaluer leurs élèves, détournant l'objectif d'apprentissage, soit construit des évaluations centrées sur les savoirs déclaratifs (définir une stratégie de lecture) et non sur les procédures (choisir une stratégie de lecture pour un projet donné) comme préconisé dans l'outil.

Enfin, un dernier élément vient renforcer la difficulté pour ces enseignants de s'approprier I'outil. II s'agit de leur entrée dans le projet. En effet, les verbalisations montrent que, malgré le vote personnel et anonyme qui leur était proposé, neuf de ces enseignants déclarent a posteriori s'être sentis, malgré tout, influencés par leurs collègues ou leur direction et avoir vécu la recherche comme une obligation.

\section{Du côté des enseignants qui s'approprient, au moins partiellement, l'outil}

Intéressons-nous maintenant aux 21 autres enseignants, qui, au terme des deux années, se sont approprié, partiellement ou totalement, l'outil proposé (12 enseignants dans le groupe «Formation » et 9 dans le groupe «Accompagnement).

Contrairement aux précédents, les autres enseignants ont vécu l'entrée dans le projet comme une démarche libre et personnelle. Ce sentiment leur a permis d'être enthousiastes et motivés par rapport à I'outil. Mais, au terme de la première journée de formation, ils découvrent l'ampleur de l'outil, les contraintes temporelles liées à la recherche et se sentent, pour certains, déstabilisés face aux démarches proposées :

Ça me semblait énorme, ça me semblait gros, j'avais l'impression qu'on n'allait pas y arriver. Et voilà, c'était trop inconnu, donc ça faisait vraiment peur. Puis après, c'était ascendant parce qu'on était quand même toutes ensemble, parce que la direction nous a quand même bien soutenus, bien encadrés (focusgroupe an 2, Astrid). 
Malgré la possibilité pour les enseignants d'effectuer certains choix, les données révèlent qu'au cours de la première année les enseignants ont implémenté l'outil en mettant en œuvre les propositions didactiques tout en restant très fidèles aux consignes et aux conseils communiqués dans le guide méthodologique. Autrement dit, ils ont respecté l'ordre des modules, utilisé la majorité des documents proposés dans l'outil, suivi les différentes consignes à l'intérieur des activités et ils ont, dans la mesure du possible, respecté les modalités de travail proposées. Le temps passé sur les activités n'a quant à lui pas pu être respecté car il avait été clairement sous-estimé par les conceptrices de l'outil.

L'année passée ont avait fait les cinq modules dans l'ordre. Cette année, on a changé l'ordre des modules et on a aussi pris plus de liberté [...]. L'année passée on a vraiment quasiment tout fait [...] selon la méthodologie du guide (entretien, Astrid).

La phase d'intégration s'observe principalement la deuxième année. Celle-ci est marquée par des transformations de l'outil. Souvent, pour une question de gestion du temps ou de complexité liée aux activités, les enseignants introduisent des variations dans la construction des synthèses, la sélection des activités et/ou des modules, le choix des supports ou du déroulement méthodologique. C'est en analysant ces transformations que l'on peut distinguer deux sous-groupes d'enseignants, en fonction de leur proximité avec les six principes didactiques sous-jacents à l'outil.

\section{Les enseignants qui s'approprient partiellement l'outil}

Au terme des deux années d'utilisation de l'outil Lirécrire, 15 enseignants (10 du groupe «Formation » et 5 du groupe «Accompagnement ») semblent avoir intégré et s'être approprié certains des principes de l'outil. C'est le cas principalement du recours aux textes informatifs et de l'enseignement explicite des stratégies de lecture. Ces enseignants ont pris conscience de la nécessité de travailler les textes informatifs dès le début du secondaire (alors qu'ils attendaient généralement la fin de la deuxième année pour les aborder). Cette appropriation cognitive s'accompagne d'un changement dans les comportements : même s'ils réduisent leur nombre (notamment lorsque la tâche porte sur des dossiers de plusieurs textes), ces enseignants utilisent les supports proposés dans l'outil ou recherchent d'autres textes à visée informative pour entraîner les apprentissages mobilisés dans l'outil Lirécrire (tels que l'entraînement des stratégies de lecture ou l'explicitation des processus de lecture ou opérations d'écriture) :

Avoir été obligée de prendre conscience que le texte informatif existait, que c'est quelque chose d'important et me rendre compte que, pour les enfants de douze ans, c'est essentiel de lire ce genre de documents, et aussi avec des techniques de lecture. C'était pas nouveau, mais je n'ai pas tendance à prendre des textes informatifs (focus-groupe an 1, Marine).

L'enseignement explicite des stratégies de lecture, que peu d'enseignants connaissaient avant leur engagement dans le projet, semble également les intéresser. Ils reconnaissent l'intérêt de la démarche, ils y voient une autre façon d'aborder le texte, moins lourde et tout aussi efficace, et ont mené la plupart des activités qui entraînent les stratégies de lecture :

Si je dois garder vraiment quelque chose de Lirécrire, moi, c'est vraiment ça. C'est dessiner la silhouette du document, je trouve que cela fonctionne vraiment très très bien, même s'ils rencontrent des difficultés pour le faire, parce qu'ils ne sont pas conscients. Mais toutes ces étapes, ces différentes stratégies, lecture survol, la lecture repérage, j'ai vraiment trouvé ça très intéressant (entretien, Émilie).

Ces enseignants disent aussi avoir pris conscience de l'intérêt de travailler à partir de situations scolaires authentiques. Ils ont principalement apprécié et mené les activités portant sur la justification, le questionnaire de lecture et la compréhension de consignes. Ils justifient leurs choix en mettant en évidence le caractère utile (directement lié aux épreuves externes de compétences soumises aux élèves) et interdisciplinaire de ces activités (qui sont sollicitées dans tous les cours et tout au long de la scolarité des élèves) :

La justification, ça me paraît primordial aussi parce qu'ils en ont tout le temps des questions avec justifications (focus-groupe an 2, Justine).

Par contre, l'entraînement "spiralaire », la pratique de la métacognition et le travail de groupe sont dévalorisés par ce groupe d'enseignants : ils ne comprennent pas l'intérêt de faire réfléchir les élèves sur leurs pratiques ou de revenir plusieurs fois sur un même texte, et ils n'hésitent pas à supprimer ces activités. Ces indices témoignent d'une faible appropriation cognitive et comportementale de ces principes particuliers.

L'analyse du parcours d'appropriation de l'outil par les enseignants permet de mettre en évidence l'existence d'obstacles liés aux contraintes temporelles de 
la recherche ou à la lassitude ressentie à certains moments. Les enseignants mettent alors en place des stratégies pour surmonter ces difficultés (se tourner vers un collègue, prendre appui sur l'accompagnement...), mais les difficultés reviennent ensuite. Dès lors, comme l'illustre l'extrait ci-dessous, ce parcours d'appropriation est fait de «hauts et de bas » et ne permet pas à l'enseignant de s'approprier l'ensemble de l'outil et de ses principes:

Les montagnes russes, ça représente bien ce que j'ai vécu. Il y a des moments où c'est chouette, on est en projet, on va apprendre plein de choses et après on est confronté à la réalité du terrain, où c'est dur pour les élèves, on a du mal à expliquer les consignes. C'est long [...], on ne sait jamais combien de temps ça va prendre (focus-groupe an 2, Sylvia).

\section{Du côté des enseignants qui s'approprient globalement l'outil}

Six enseignants (2 dans le groupe «Formation » et 4 dans le groupe "Accompagnement») témoignent d'une appropriation beaucoup plus complète de l'outil. En effet, au-delà d'une valorisation discursive (appropriation cognitive) et comportementale des principes didactiques relatifs à l'enseignement explicite des stratégies de lecture des textes informatifs, ils paraissent s'être également approprié les autres principes didactiques de l'outil.

Le fait d'aider les élèves à verbaliser leurs démarches de lecteurs-scripteurs et leurs difficultés de lecture au moyen d'activités de métacognition planifiées et organisées (par le recours notamment aux grilles d'analyse de ses pratiques proposées dans l'outil) semble constituer une nouvelle pratique pédagogique susceptible d'être utilisée par ces enseignants :

Tout ce qui est métacognitif aussi. [...] Réfléchir à la manière dont eux-mêmes conçoivent leurs stratégies. C'est hyper intéressant pour eux. Ce n'est jamais fait donc j'aime bien justement cette manière un peu décalée de voir les choses (entretien, Arthur).

Même si au départ il a pu déstabiliser ces enseignants, l'entraînement « spiralaire » semble également les intéresser :

Je suis d'accord que c'est parfois un peu long de rester sur un même texte, mais j'ai vraiment l'impression que, pour bien comprendre la stratégie, il faut rester dans un seul texte. Sinon, pour nous adultes, on peut très bien travailler les stratégies de lecture avec différents textes, mais les élèves ont besoin qu'on fasse ça avec un seul parce que, sinon, ils sont trop absorbés par le contenu plutôt que par la démarche (focus-
Enfin, malgré les difficultés rencontrées dans la gestion de leurs classes, certains enseignants ont découvert l'intérêt de varier les modalités de travail et, dans certains cas, ont mis en place une «charte du bon fonctionnement du travail en sous-groupes», ce qui leur a permis d'y accorder plus d'importance:

Ce qui m'intéresse le plus dans ce programme c'est le travail en groupe, en sous-groupes. J'ai davantage travaillé en groupe parce que j'avais une classe qui nécessitait qu'on travaille en groupe [...]. Maintenant, il fallait que je contrôle plus le travail. Mais leur donner une tâche définie à faire, dans un laps de temps défini, leur permettait de se sentir beaucoup plus à l'aise et d'être moins encerclés par le contexte scolaire (focusgroupe an 2, Arthur).

Ces enseignants n'hésitent pas à prolonger les activités de l'outil avec d'autres tâches (diffusion d'une émission portant sur un des sujets repris dans l'outil, lecture d'un roman en guise de prolongement...) et à reprendre certaines de ces démarches dans leurs autres cours (de français ou autres). Le transfert semble se faire progressivement, mais un suivi de l'usage de l'outil à plus long terme devrait être mené pour pouvoir confirmer cette observation. De plus, d'après leurs témoignages, et à la différence des autres enseignants, ceux-ci ont le sentiment de s'être approprié l'outil, «petit à petit », « marche après marche». Les difficultés ressenties à certains moments ont pu être franchies progressivement et tendent à disparaître avec le temps.

Ces enseignants se posent également beaucoup de questions, s'interrogent sur leurs pratiques et sur les fondements théoriques et méthodologiques de I'outil. Ils trouvent notamment, pour certains d'entre eux, leurs réponses lors des séances d'accompagnement qui sont proposées :

Les pointillés représentent les moments d'interrogation, de flou, de latence mais qui finalement repartent vers le haut parce qu'on est une équipe assez soudée. Et du coup, dans les moments de latence, ça repart en positif parce qu'on retrouve quand même des pistes et des solutions (focus-groupe an 2, Astrid).

\section{Facteurs associés à l'appropriation de l'outil Lirécrire}

Plusieurs facteurs liés à la recherche, à l'outil, à la classe, aux enseignants et à l'environnement organisationnel sont associés au processus d'appropriation de l'outil didactique. Ces facteurs semblent agir différemment 
d'une année à l'autre. Cette distinction sera donc prise en compte dans l'analyse qui suit.

La première année, la mise en œuvre semble surtout facilitée par des facteurs liés à l'outil et au dispositif de recherche. Du côté de l'outil, en dépit de la densité des informations contenues dans le guide méthodologique (c'est par ce support que les enseignants ont accès aux conduites de classe détaillées, aux corrigés des exercices, aux conseils méthodologiques...), les enseignants le consultent souvent pour mettre en œuvre les activités proposées. II a principalement été utilisé pour, d'une part, préparer et implémenter le plus fidèlement possible les activités et, d'autre part, mener les mises en commun et les corrections d'exercices :

Moi, ça m'a rassuré, le guide méthodologique. Justement parce que c'était «téléguidé». Donc, on ne nous lâchait pas dans la nature [...]. Moi, le guide, le manuel, je ne l'ai presque pas regardé cette année. Tandis que, l'année dernière, c'était ma Bible, mon livre de chevet. Ici, j'étais très content d'avoir ce manuel (focus-groupe an 2, Arthur).

Néanmoins, certains aspects matériels et pédagogiques liés à l'outil freinent l'appropriation : pour quelques enseignants, les trois volumes complexifient la manipulation en classe, l'entrée par le guide méthodologique empêche d'avoir une vision globale sur les activités, la sous-estimation temporelle de certaines activités rend difficile la planification didactique, etc.

En relation avec le dispositif de recherche, les enseignants déclarent que les moments de formation et d'accompagnement les ont aidés à s'approprier l'outil. Plus précisément, ils rapportent que les journées de formation leur ont permis de découvrir les principaux fondements théoriques de l'outil, de comprendre sa structure et la façon de manipuler les trois volumes de l'outil. Les séances d'accompagnement les ont aidés à mieux diagnostiquer les difficultés des élèves, à planifier les activités de l'outil (en se fixant des objectifs précis), à échanger à propos de leurs pratiques de classe et à trouver des réponses à des questions liées à leurs pratiques pédagogiques ou leur mise en œuvre de l'outil. La majorité des enseignants du groupe «Accompagnement » déplore d'ailleurs l'absence d'accompagnement au cours de la deuxième année :

Les séances d'accompagnement c'était un moment où on reprenait conscience de l'intérêt du projet (entretien, Laetitia).

Du côté des facteurs liés à l'environnement organisationnel, de manière un peu surprenante, les enseignants du groupe «Formation» paraissent avoir développé davantage de pratiques collaboratives (formelles ou informelles) que ceux du groupe «Accompagnement». Cette collaboration semble se poursuivre tout au long des deux années d'expérimentation de l'outil («Au moins une fois par semaine, on prend un temps pour parler de ça, on retravaille tout, [...] on donne exactement la même chose», entretien, Fiona) alors qu'elle disparaît la seconde année dans le groupe «Accompagnement». Les caractéristiques liées à la recherche expliquent peut-être cette différence. En effet, les données collectées permettent de constater que les enseignants du groupe «Accompagnement » se sont principalement appuyés sur les trois séances d'accompagnement pour travailler et réfléchir à propos de l'outil Lirécrire. Autrement dit, ces enseignants ont compté sur les chercheurs pour échanger ou partager les supports didactiques, planifier les activités, se rassurer face aux difficultés rencontrées, etc. Ces enseignants ne se sont plus réunis la deuxième année pour échanger à propos de l'outil. Pour certains, cette absence de collaboration a eu un impact sur leur motivation à mettre en œuvre l'outil la deuxième année. Les enseignants du groupe «Formation» ont quant à eux développé des stratégies de soutien à la mise en œuvre en planifiant notamment, dès la première année, des réunions de travail régulières ou en se réunissant de façon plus informelle, sur le temps de midi. En effet, malgré l'absence $d^{\prime}$ heure de coordination ou de soutien de la direction dans ces écoles, deux établissements ont pris l'initiative de préparer et d'organiser des réunions de travail (une fois par semaine ou à certains moments-clés dans l'année : en début d'année, avant les examens...) pour planifier les activités de l'outil, pour retravailler certains supports (les synthèses notamment) ou, tout simplement, pour échanger à propos de leurs difficultés :

On se voyait presque une fois par semaine, mais ça ne durait pas très longtemps puisqu'on avait préparé au préalable chacune de notre côté et puis on se mettait d'accord, on regardait si on avait sélectionné les mêmes activités, et si dans les activités on faisait tous les points (focus-groupe an 1, Valentine).

Dans les trois autres écoles appartenant au groupe "Formation», ces échanges de documents ou de conseils autour de l'outil se sont principalement concrétisés lors de moments plus informels (à la récréation, durant les heures de battement communes ou sur le temps de midi).

Dans tous les cas, ces moments d'échange et de partage ont été ressentis par les enseignants comme 
un soutien à leur motivation et leur engagement, mais également comme une aide à la compréhension des principes de l'outil :

Les fois où on a travaillé entre nous, c'était beaucoup plus facile, on partage nos expériences, dire « moi j'ai fait comme ça, ah c'est une bonne idée», et tous des trucs comme ça, ça a vraiment facilité la mise en œuvre (focus-groupe an 1, Justine).

La première année, les contraintes temporelles liées à la recherche ont représenté un facteur défavorable à l'appropriation de l'outil. En effet, entre le moment où les enseignants ont reçu l'outil et ont participé aux deux journées de formation, la majorité des équipes éducatives a réellement mis en œuvre les activités au mois de janvier. Or les passations d'épreuves de compétences nécessitaient que les enseignants aient terminé au mois de mai, soit cinq mois plus tard. Tous ont ressenti une certaine pression temporelle :

L'année dernière, j'ai tout fait. On n'avait presque pas de temps, donc j'ai foncé vers le manuel et j'ai suivi les lignes. Ce qu'on devait faire, du début à la fin (focus-groupe an 2, Elsa).

Nos analyses montrent également que les pratiques et les conceptions antérieures des enseignants liées à l'enseignement de la lecture et de l'écriture ont aussi des répercussions sur la façon de s'approprier l'outil. Les enseignants dont les pratiques ou les conceptions sont les plus éloignées de l'outil sont également ceux dont l'appropriation apparaît faible, voire nulle. Leurs conceptions et pratiques affectent leur rapport global à l'outil : difficulté de maintenir leur motivation, incompréhension ou désaccord menant à une suppression de certaines démarches proposées dans l'outil (activités de métacognition, travail de groupe...), détournement des objectifs d'apprentissage décrits dans l'outil (création d'évaluations centrées sur les savoirs et non sur les démarches, centration sur les aspects liés à la langue...).

Relevons enfin que des dimensions liées à la classe semblent représenter un frein potentiel pendant la première année (à propos de la réalisation de travaux de groupes, par exemple) et un adjuvant pendant la seconde année. En effet, la réaction positive des élèves face aux activités et la perception de l'évolution de certains élèves paraissent jouer un rôle important dans l'appropriation de l'outil par les enseignants et les encourager à s'engager davantage dans celle-ci.

\section{Discussion et conclusion}

L'objectif de cet article est double : analyser le processus d'appropriation d'un outil didactique par les enseignants et identifier le rôle joué dans ce processus par une diversité de facteurs. Pour caractériser ce processus, nous nous sommes appuyés sur le modèle proposé par de Vaujany (2006), que nous avons précisé et élargi pour l'adapter à notre objet d'étude.

Rappelons que cette étude s'appuie sur des données collectées auprès de 35 enseignants volontaires qui ont participé au projet de recherche. Malgré l'absence d'observations dans les classes, ce qui constitue une limite de notre travail, nos analyses font apparaître, pour un peu plus de la moitié des enseignants suivis, de nombreux indices d'un processus d'appropriation de l'outil, conduisant, au moins partiellement, à un changement de conceptions et de pratiques d'enseignement de la lecture dans le sens valorisé par l'outil.

C'est en particulier le cas pour les deux premiers principes sous-jacents à la proposition Lirécrire, à savoir la place accordée aux textes informatifs et à l'enseignement explicite des stratégies de lecture. Quelques enseignants ont accordé davantage de place aux activités authentiques, aux activités menées en sousgroupes, aux activités de métacognition, et à l'enseignement «spiralaire», mais globalement, on constate une distinction nette entre l'accueil des deux premiers principes, visiblement plus favorable, et celui des quatre autres que les enseignants peinent davantage à intégrer dans leurs pratiques.

II nous semble à cet égard important de relever que les deux principes les plus largement acceptés par les enseignants renvoient à une modification de leurs propres pratiques (le type de texte choisi et les consignes de travail permettant un enseignement explicite des stratégies de lecture), sans pour autant modifier significativement les situations d'enseignement et les rôles respectifs des enseignants et des élèves au sein de la classe. Plusieurs des principes gardés à distance par les enseignants, ou faisant l'objet de turbulences lors de leur mise en œuvre, touchent plus fondamentalement les situations de classe et, dans une certaine mesure, la forme scolaire (Vincent, 1994). Par exemple, travailler sur des situations authentiques conduit à sortir de la segmentation traditionnelle des savoirs sur laquelle repose la forme scolaire; travailler en sous-groupes dans une perspective d'apprentissage coopératif induit d'autres relations entre les élèves, ainsi qu'entre les élèves et I'enseignant. Nous faisons I'hypothèse qu'une partie des 
difficultés à surmonter pour l'appropriation d'un tel outil dépasse des questions strictement didactiques et révèle des obstacles plus fondamentaux, liés à la perception de ce qu'est l'école et de ce que peuvent recouvrir des situations d'enseignement en classe.

Soulignons également que les obstacles à l'appropriation et les stratégies de contournement observés face à l'outil proposé convergent avec les résultats de recherches antérieures, révélant que le changement de pratiques pédagogiques est un processus long et difficile. La méta-analyse menée par l'équipe de Borman (2003) montre notamment que les enseignants peuvent avoir besoin de temps (jusqu'à cinq années d'usage d'un outil) pour dépasser les difficultés rencontrées dans la mise en œuvre de nouvelles propositions didactiques. Ce processus d'appropriation est donc progressif et s'inscrit dans la durée (Cèbe \& Goigoux, 2007; Duthoit \& Metz, 2012). Nos propres résultats montrent que la première année d'appropriation d'un outil d'une telle ampleur est surtout orientée vers le respect des consignes : la plupart des processus d'ajustement et de transformation de l'outil ne sont en effet observés que lors de la deuxième année de la recherche. L'implémentation de l'outil didactique, c'est-à-dire sa mise en œuvre conforme aux prescriptions, semble ainsi représenter une étape nécessaire à son appropriation, mais elle ne garantit pas la transition vers son intégration personnelle.

Nos résultats semblent soutenir l'idée que plusieurs facteurs liés à la recherche, à l'outil, à la classe et à l'environnement organisationnel interviennent à des degrés divers dans le processus d'appropriation (Bélanger, Bowen, Cartier et al., 2012; Dupriez, 2015; Horn \& Little, 2010). Ils font ainsi apparaître l'intérêt d'un guide méthodologique détaillé, source de sécurité pour les enseignants, et les influences contrastées que les classes peuvent avoir sur l'usage et l'appropriation de l'outil par les enseignants. Ils mettent également en évidence les difficultés rencontrées par certains enseignants dont les pratiques antérieures sont fortement distantes avec celles suggérées par l'outil proposé.

La littérature scientifique souligne également l'importance de la collaboration entre enseignants comme soutien à l'appropriation d'outils didactiques (Dupriez, 2015; Horn \& Little, 2010; Little, 2006). Mais, d'après Horn et Little (2010), pour qu'elle amène à un déplacement ou à un ajustement de pratiques pédagogiques, cette collaboration doit permettre aux équipes éducatives de partager leurs pratiques de classe et de centrer leurs discussions sur l'apprentissage des élèves. On comprend mieux le succès de la formation et de l'accompagnement construits dans le cadre de la recherche Lirécrire, qui sont perçus comme des adjuvants importants par tous les enseignants de l'échantillon. Si la formation les a amenés à parler de leurs premières impressions et expériences à propos de l'outil à mettre en œuvre (en termes de manipulation et de planification), elle a également permis de mettre au cœur de la discussion l'élève, ses difficultés de lecture et d'écriture et les stratégies qu'il met généralement en place (en explicitant les fondements théoriques de l'outil). Du côté de l'accompagnement, c'est au travers de l'analyse des traces apportées par les enseignants (activités complétées, productions écrites...) que ceux-ci ont pu identifier les leviers et les obstacles à la mise en œuvre des activités et partager à propos des apprentissages de leurs élèves.

Il est toutefois à la fois intéressant et à première vue surprenant de constater que la collaboration entre les enseignants d'une même école participant à la recherche au cours de la seconde année de travail apparaît comme étant plus solide au sein des équipes éducatives du groupe "Formation» que du groupe «Accompagnement». II convient d'être prudent dans l'interprétation de cette différence. Mais, malgré le petit nombre d'équipes éducatives concernées, il nous semble important de souligner que les données à notre disposition suggèrent que cette différence n'est pas liée à ce qui préexistait au sein de ces établissements, mais plutôt au processus d'implication dans la recherche. En quelque sorte, les enseignants «accompagnés » au cours de la première année s'en remettent aux chercheurs pour organiser et structurer leurs échanges, mais le départ des chercheurs (la seconde année) ne permet pas à ces équipes de construire un scénario de travail collaboratif alternatif. Au contraire, dans le groupe «Formation", l'insécurité générée par le dispositif de recherche conduit ces enseignants, dès la première année, à s'auto-organiser collectivement. Et dans certaines écoles, ces dispositifs mi-formels, miinformels subsistent et permettent de soutenir les enseignants lors de la seconde année.

Contrairement à d'autres études (Spillane, Halverson \& Diamond, 2000), le soutien de la direction ne semble pas être un facteur déterminant pour l'appropriation de l'outil. Ce résultat peut faire l'objet de diverses interprétations. D'une part, après un accord de la direction pour travailler avec leur établissement, ce projet s'adressait spécifiquement et directement aux professeurs de français et c'est à cette échelle que 
le travail était planifié et soutenu. Le dispositif de travail mis en place octroyait donc a priori peu de place à la direction. D'autre part, des recherches antérieures ont montré que, dans l'enseignement secondaire belge francophone, les directions d'établissement ont habituellement peu de temps à consacrer aux questions pédagogiques (Desmet, Dupriez \& Galand, 2017).

Enfin, dans la perspective des travaux de Cèbe et Goigoux (2007), qui conçoivent l'appropriation d'outils didactiques comme un «processus de conception continuée dans l'usage», faisant de l'outil didactique un vecteur de changement de pratiques pédagogiques et de développement professionnel, on peut, globalement, s'interroger sur la capacité du dispositif Lirécrire à faciliter de telles évolutions des pratiques pédagogiques. En effet, si un outil didactique doit être cohérent avec les résultats de la recherche, il doit aussi être en proximité suffisante avec les pratiques habituelles des enseignants. La recherche présentée dans cet article met en évidence une distance parfois grande entre les pratiques des enseignants et les activités proposées par Lirécrire. Si tous les enseignants ont accepté de mettre en œuvre la majorité des activités proposées lors de la première année, pour près de la moitié d'entre eux, cet outil est source d'une grande déstabilisation, les conduisant à rapidement se désengager. II y a au minimum ici une tension, redoutablement complexe, entre la volonté de promouvoir le changement et la nécessité, semble-t-il, de ne pas trop s'éloigner des pratiques habituelles afin de ne pas perdre trop d'enseignants en cours de route. Ces constatations nous éclairent également sur la façon de concevoir les outils didactiques : une analyse préalable du travail de l'enseignant et des modifications, des efforts ou des renoncements qu'impliquera l'usage de cet outil par les enseignants est nécessaire. Au regard des conclusions issues de nos analyses, l'outil Lirécrire pour apprendre sera quant à lui modifié. Ces modifications structurelles (public élargi, manipulation plus efficace...), didactiques et pédagogiques (mise à disposition d'outils d'observation des élèves, soutien à la mise en œuvre des étapes-clés, explicitation des objets à enseigner...) tiendront compte des contraintes explicitées par les enseignants et viseront à s'ajuster davantage à leurs pratiques. La présente recherche souligne également tout l'intérêt des dispositifs de formation et d'accompagnement pensés comme des situations de formation «à la pratique et par la pratique », très appréciés par les enseignants. Le dispositif d'accompagnement en particulier semble très utile pour faciliter l'appropriation, c'est-à-dire une intégration personnelle de l'outil, pouvant conduire à certaines transformations de celui-ci. Rappelons par ailleurs, tout en restant très prudents au regard de la petite taille de l'échantillon, que la grande majorité des enseignants du groupe «Accompagnement » se sont approprié l'outil, au moins partiellement. Cela nous pousse à penser que ce dispositif d'accompagnement est pertinent, qu'il devrait probablement être prolongé au-delà de la première année et qu'il devrait aussi veiller à préparer la transition, en mettant en place les bases d'un dispositif local de travail collaboratif entre enseignants qui se poursuivra au-delà de la présence des chercheurs.

Jessica Penneman Université catholique de Louvain jessica.penneman@uclouvain.be

Séverine De Croix

Université catholique de Louvain Haute École Léonard de Vinci

Sébastien Dellisse

Université catholique de Louvain

Jean-Louis Dufays

Université catholique de Louvain

Xavier Dumay

Université catholique de Louvain

Vincent Dupriez

Université catholique de Louvain

Benoît Galand

Université catholique de Louvain

Marielle Wyns

Université catholique de Louvain Haute École Léonard de Vinci 


\section{Bibliographie}

BAUTIER É. \& GOIGOUX R. (2004). «Difficultés d'apprentissage, processus de secondarisation et pratiques enseignantes : une hypothèse relationnelle». Revue française de pédagogie, $\mathrm{n}^{\circ} 148$, p. 89-100.

BÉLANGER J., BOWEN F., CARTIER S., DESBIENS N., MONTÉSINOS-GELET I. \& TURCOTTE L. (2012). «L'appropriation de nouvelles pratiques d'interventions pédagogiques et éducatives en milieu scolaire : réflexions sur un cadre théorique intégrateur ». Éducation et francophonie, vol. 40, $n^{\circ} 1$, p. 56-75.

BERMAN P. (1980). «Thinking about programmed and adaptive implementation: Matching strategies to situations». In H.M. Ingram \& D. E. Mann (dir.), Why policies succeed or fail. Beverly Hills : Sage, p. 205-228.

BIESTA G. (2007). «Why "what works" won't work: Evidencebased practice and the democratic deficit in educational research ». Educational theory, vol. 57, n 1 , p.1-22.

BONNÉRY S. (2015). Supports pédagogiques et inégalités scolaires. Paris : La Dispute.

BORMAN G. D., HEWES G. M., OVERMAN L. T. \& BROWN S. (2003). «Comprehensive school reform and achievement: A meta-analysis ». Review of Educational Research, vol. 2, $\mathrm{n}^{\circ} 73, \mathrm{p} .125-230$.

BUCHETON D. (2014). Refonder l'enseignement de l'écriture : vers des gestes professionnels plus ajustés du primaire au lycée. Paris : Retz.

CARTIER S., CONTANT H. \& JANOSZ M. (2012). «Appropriation de pratiques pédagogiques sur l'apprentissage par la lecture en classe de français du secondaire en milieu défavorisé au Québec ». Repères, n²5, p. 97-115.

CÈBE S. \& GOIGOUX R. (1999). "L'influence des pratiques d'enseignement sur les apprentissages des élèves en difficulté ». Cahiers Alfred Binet, vol. 661, n4, p. 49-68.

CÈBE S. \& GOIGOUX R. (2007). "Concevoir un instrument didactique pour améliorer l'enseignement de la compréhension de textes ». Repères, n³5, p. 185-208.

CÈBE S. \& GOIGOUX R. (2009). Lector et Lectrix : apprendre à comprendre les textes narratifs. Paris : Retz.

CHARLIER É. \& BIÉMAR S. (2012). Accompagner : un agir professionnel. Bruxelles : De Boeck.

CHARTIER A.-M. (2000). «Réussite, échec et ambivalence de l'innovation pédagogique : le cas de l'enseignement de la lecture ». Recherche \& formation, $n^{\circ} 34$, p. 41-56.

CORRIVEAU L., LETOR C. \& BAGNOUD D. P. (2010). Travailler ensemble dans les établissements scolaires et de formation: processus, stratégies, paradoxes. Bruxelles : De Boeck.

CRINON J. \& MARIN B. (2008). Apprendre à écrire des textes explicatifs en situation de révision collaborative. Papier présenté dans le cadre du Colloque international «De la France au Québec : L'Écriture dans tous ses états», Poitiers.

DE CROIX S. \& PENNEMAN J. (2014). Lirécrire pour apprendre. Comprendre les textes informatifs. Louvain-la-Neuve : Uni- versité catholique de Louvain et Fondation Louvain.

DE CROIX S., PENNEMAN J. \& WYNS M. (2017). «Concevoir un outil didactique pour enseigner la lecture des textes informatifs au début du secondaire». In M. Brunel, J. Émery-Bruneau, J.-L. Dufays, O. Dezutter \& É. Falardeau (dir.), L'enseignement et l'apprentissage de la lecture aux différents niveaux de la scolarité. Namur : Presses universitaires de Namur, Diptyque, $\mathrm{n}^{\circ} 35$.

DE VAUJANY F. X. (2006). «Pour une théorie de l'appropriation des outils de gestion : vers un dépassement de l'opposition conception-usage ». Management \& Avenir, vol. 3, $n^{\circ} 9$, p. 109-126.

DESIMONE L. (2002). "How can comprehensive school reform models be successfully implemented?». Review of Educational Research, vol. 72, n³, p. 433-479.

DESMET L., DUPRIEZ V. \& GALAND B. (2017). «Que font les écoles accueillant des élèves défavorisés des moyens supplémentaires qui leur sont alloués? Une étude longitudinale de cinq établissements scolaires en encadrement différencié». Les Cahiers de recherche du Girsef, n 108.

DUPRIEZ V. (2015). Peut-on réformer l'école? Approches organisationnelle et institutionnelle du changement pédagogique. Louvain-la-Neuve : De Boeck.

DURLAK J. A. \& DUPRE E. P. (2008). «Implementation matters: A review of research on the influence of implementation on program outcomes and the factors affecting implementation ». American journal of community psychology, vol. 3-4, n 41 , p. 327-350.

DUSENBURY L., BRANNIGAN R., FALCO M. \& HANSENW. B. (2003). «A review of research on fidelity of implementation: implications for drug abuse prevention in school settings». Health education research, vol. $18, \mathrm{n}^{\circ} 2$, p.237-256.

DUTHOIT E. \& METZ S. (2012). «Analyse de l'appropriation d'un parcours pédagogique numérique par un formateur : le cas du dispositif Pairformance». Activité, vol. 1, $n^{\circ} 9$, p. 106-126.

FALARDEAU É. \& GAGNÉ J.-C. (2012). «L'enseignement explicite des stratégies de lecture: des pratiques fondées par la recherche». Enjeux, n83, p. 91-120.

FULLAN M. (2015). The New Meaning of Educational Change. New York : Teacher's College Press.

GOIGOUX R. (2007). «Un modèle d'analyse de l'activité des enseignants». Éducation et didactique, vol. 1, n³, p. 47-69.

HORN I. S. \& LITTLE J. W. (2010). «Attending to Problems of Practice: Routines and Resources for Professional Learning in Teachers' Workplace Interactions ». American Educational Research Journal, vol. 47, ${ }^{\circ} 1$, p.181-217.

JACOBS V. A. \& IPPOLITO J. (2015). «Improving comprehension of informational texts in the secondary classroom ». In S. R. Parris \& K. Headley (dir.), Comprehension Instruction: Research-Based Best Practices. Londres : The Guilford Press, p. 278-290. 
JANOSZ M. \& DENIGER M. (2001). Évaluation de programmes de prévention du décrochage scolaire pour adolescents de milieux défavorisés. Montréal : Centre de recherche et d'intervention sur la réussite scolaire (CRIRES) et Institut de recherche pour le développement social des jeunes (IRDS).

JANOSZ M., GEORGES P. \& PARENT S. (1998). «L'environnement socioéducatif à l'école secondaire : un modèle théorique pour guider l'évaluation du milieu ». Revue canadienne de psycho-éducation, vol. 27, n² 2, p. 285-306.

LANGENBERG D. N. (2000). Report of the national reading panel. Teaching children to read: An evidence-based assessment of the scientific research literature on reading and its implications for reading instruction: Reports of the subgroups. Bethesda : National Institute of Child Health and Human Development, National Institutes of Health.

LETOR C. (2009). Comment travailler en équipe dans les établissements scolaires? Bruxelles : De Boeck.

LITTLE J.W. (2006). Professional development and professional community in the learner-centered school. Washington : National Education Association.

MÄRZ V. (2014). «It takes two to tango »: Structuring actors and acting structures in the implementation of educational innovations. Thèse de doctorat, sciences de l'éducation, Université de Leuven, Faculté de psychologie et des sciences de l'éducation, Centre for Innovation and the Development of Teacher and School.

NONNON É. (2012). «Dimension épistémique de la lecture et construction de connaissances à partir de l'écrit : enjeux, obstacles, apprentissages ». Repères, $n^{\circ}$ 45, p.7-37.

PAO L. S. \& WILLIAMS J. P. (2015). «Improving Comprehension of Informational Texts in the Elementary Classroom ». In S. R. Parris \& K. Headley (dir.), Comprehension Instruction: Research-Based Best Practices. Londres : The Guilford Press, p. 238-252.
RINGWALT C. L., ENNETT S., JOHNSON R., ROHRBACH L. A., SIMONS-RUDOLPH A., VINCUS A. \& THORNE J. (2003). «Factors Associated With Fidelity to Substance Use Prevention Curriculum Guides in the Nation's Middle Schools». Health Education \& Behavior, vol. 30, n³, p.375-391.

ROWAN B. \& MILLER R. J. (2007). «Organizational strategies for promoting instructional change: Implementation dynamics in schools working with comprehensive school reform providers ». American Educational Research Journal, vol. 44, n², p. 252-297.

SNOW C. (2002). Reading for understanding: Toward an R\&D program in reading comprehension. Santa Monica : Rand Corporation.

SPILLANE J. P., HALVERSON R. \& DIAMOND J. (2000). Toward a theory of leadership practice: A distributed leadership perspective. Papier présenté dans le cadre de la Conférence annuelle de l'Association américaine de recherche en éducation, New Orleans.

SPILLANE J. P., REISER B. \& REIMER T. (2002). «Policy Implementation and Cognition : Reframing and Refocusing Implementation Research». Review of Educational Research, vol. 72, n³, p. 387-431.

THEUREAU J. (2011). Appropriation 1, 2, 3 ou Appropriation, Incorporation «Inculturation». Papier présenté dans le cadre de la Journée Ergo-Idf Appropriation \& Ergonomie, Paris.

VAN GRUNDERBEECK N., THÉORÊT M., CHOUINARD R. \& CARTIER S. (2004). Suggestions de pratiques d'enseignement favorables au développement de la lecture chez les élèves du secondaire. Montréal : Université de Montréal.

VINCENT G. (1994). L'éducation prisonnière de la forme scolaire? Scolarisation et socialisation dans les sociétés industrielles. Lyon : Presses universitaires de Lyon. 


\begin{tabular}{|c|c|c|}
\hline $\begin{array}{l}\text { Principes à } \\
\text { s'approprier }\end{array}$ & $\begin{array}{l}\text { Dimension de } \\
\text { l'appropriation }\end{array}$ & Indicateurs observables \\
\hline \multirow[t]{3}{*}{$\begin{array}{l}\text { Textes } \\
\text { informatifs }\end{array}$} & $\begin{array}{l}\text { Appropriation } \\
\text { cognitive }\end{array}$ & $\begin{array}{l}\text { Comment l'enseignant parle-t-il des textes informatifs? } \\
\text { Exemple : l'enseignant dit qu'il a pris conscience de la nécessité de travailler sur } \\
\text { des supports et des documents à visée informative dès l'entrée dans le secon- } \\
\left.\text { daire (en } 1^{\text {re }}\right)\end{array}$ \\
\hline & \multirow[t]{2}{*}{$\begin{array}{l}\text { Appropriation } \\
\text { comportementale }\end{array}$} & $\begin{array}{l}\text { Quels supports/documents appartenant à l'outil l'enseignant utilise-t-il? } \\
\text { Exemple: l'enseignant utilise ou sélectionne les supports/documents de l'outil }\end{array}$ \\
\hline & & $\begin{array}{l}\text { L'enseignant construit-il d'autres supports/documents que ceux proposés } \\
\text { dans l'outil? } \\
\text { Exemple : l'enseignant construit de nouvelles activités (d'évaluation, d'appren- } \\
\text { tissage ou d'entraînement) à partir de supports/documents de l'outil ou d'autres } \\
\text { supports/documents à visée informative, proches de la réalité scolaire des } \\
\text { élèves (manuels, questionnaires, textes documentaires) que ceux proposés dans } \\
\text { l'outil }\end{array}$ \\
\hline \multirow[t]{3}{*}{$\begin{array}{l}\text { Stratégies de } \\
\text { lecture }\end{array}$} & $\begin{array}{l}\text { Appropriation } \\
\text { cognitive }\end{array}$ & $\begin{array}{l}\text { Comment l'enseignant parle-t-il de l'enseignement des stratégies de lec- } \\
\text { ture? } \\
\text { Exemple: l'enseignant prend conscience de l'importance de faire expliciter les } \\
\text { stratégies de lecture lors des activités centrées sur la lecture de textes }\end{array}$ \\
\hline & \multirow[t]{2}{*}{$\begin{array}{l}\text { Appropriation } \\
\text { comportementale }\end{array}$} & $\begin{array}{l}\text { Dans quelle mesure l'enseignant met-il en œuvre les activités de l'outil por- } \\
\text { tant sur l'explicitation des stratégies de lecture? } \\
\text { Exemple : l'enseignant met en œuvre toutes les activités de l'outil portant sur } \\
\text { l'explicitation des stratégies de lecture }\end{array}$ \\
\hline & & $\begin{array}{l}\text { Dans quelle mesure l'enseignant construit-il des nouveaux outils (activités, } \\
\text { supports, synthèses) portant sur l'explicitation ou l'entraînement des straté- } \\
\text { gies de lecture inspirées/proches de celles proposées dans l'outil (à des fins } \\
\text { d'évaluation, d'apprentissage ou d'entraînement)? }\end{array}$ \\
\hline \multirow[t]{5}{*}{$\begin{array}{l}\text { Entraînement } \\
\text { «spiralaire" }\end{array}$} & \multirow[t]{2}{*}{$\begin{array}{l}\text { Appropriation } \\
\text { cognitive }\end{array}$} & $\begin{array}{l}\text { Que pense l'enseignant à propos de la répétition des démarches? } \\
\text { Exemple: l'enseignant estime qu'il est nécessaire d'entraîner plusieurs fois les } \\
\text { stratégies de lecture pour qu'elles soient maîtrisées par les élèves }\end{array}$ \\
\hline & & Que pense l'enseignant de la progression des activités de l'outil? \\
\hline & \multirow[t]{3}{*}{$\begin{array}{l}\text { Appropriation } \\
\text { comportementale }\end{array}$} & $\begin{array}{l}\text { Dans quelle mesure l'enseignant suit-il la progression proposée en entraî- } \\
\text { nant les stratégies à plusieurs reprises? } \\
\text { Exemple : l'enseignant suit l'ordre des activités proposées } \\
\text { Contre-exemple: dans la sélection des activités relatives aux stratégies de lec- } \\
\text { ture, l'enseignant fusionne certaines activités }\end{array}$ \\
\hline & & Dans quelle mesure l'enseignant met-il en œuvre les tâches d'intégration? \\
\hline & & $\begin{array}{l}\text { Dans quelle mesure l'enseignant met-il en œuvre les activités de } \\
\text { prolongement? }\end{array}$ \\
\hline \multirow[t]{2}{*}{ Mode de travail } & $\begin{array}{l}\text { Appropriation } \\
\text { cognitive }\end{array}$ & $\begin{array}{l}\text { Que pense l'enseignant du travail de groupe? } \\
\text { Exemple : l'enseignant prend conscience de l'apport du travail de groupe (par } 2 \\
\text { ou plus) }\end{array}$ \\
\hline & $\begin{array}{l}\text { Appropriation } \\
\text { comportementale }\end{array}$ & $\begin{array}{l}\text { Dans quelle mesure l'enseignant met-il en œuvre les activités de l'outil en } \\
\text { sous-groupes? }\end{array}$ \\
\hline
\end{tabular}




\begin{tabular}{l|l|l}
\hline $\begin{array}{l}\text { Principes à } \\
\text { s'approprier }\end{array}$ & $\begin{array}{l}\text { Dimension de } \\
\text { l'appropriation }\end{array}$ & Indicateurs observables \\
\hline Métacognition & $\begin{array}{l}\text { Appropriation } \\
\text { cognitive }\end{array}$ & $\begin{array}{l}\text { Que pense l'enseignant des activités de métacognition? } \\
\text { Exemple : l'enseignant prend conscience de l'apport des grilles d'analyse des } \\
\text { pratiques et des retours réflexifs proposés dans l'outil. }\end{array}$ \\
\cline { 2 - 3 } & $\begin{array}{l}\text { Appropriation } \\
\text { comportementale }\end{array}$ & $\begin{array}{l}\text { Dans quelle mesure l'enseignant mène-t-il les activités métacognitives de } \\
\text { l'outil avec supports (grilles d'analyse des pratiques et conseils, bonnes } \\
\text { démarches)? }\end{array}$ \\
\cline { 2 - 3 } & $\begin{array}{l}\text { Dans quelle mesure l'enseignant mène-t-il les activités métacognitives sans } \\
\text { supports («retours réflexifs »)? }\end{array}$ \\
\hline \multirow{2}{*}{$\begin{array}{l}\text { Situations } \\
\text { authentiques }\end{array}$} & $\begin{array}{l}\text { Appropriation } \\
\text { cognitive }\end{array}$ & $\begin{array}{l}\text { Que pense l'enseignant des activités portant sur la création de cartes men- } \\
\text { tales, de questionnaires, de justifications...? } \\
\text { Exemple: l'enseignant trouve le travail sur les cartes mentales, tables des } \\
\text { matières, questionnaires, justifications... intéressant }\end{array}$ \\
\cline { 2 - 3 } & $\begin{array}{l}\text { Appropriation } \\
\text { comportementale }\end{array}$ & $\begin{array}{l}\text { Dans quelle mesure l'enseignant met-il en œuvre les activités proches de la } \\
\text { réalité scolaire des élèves : création de cartes mentales, création du ques- } \\
\text { tionnaire de lecture, production de questions ou de justifications...? }\end{array}$ \\
\hline
\end{tabular}

Annexe 2. Grille d'analyse des facteurs impliqués dans le processus d'appropriation de l'outil Lirécrire pour apprendre

\begin{tabular}{l|l}
\hline \multirow{2}{*}{ Facteurs } & Dimensions analysées \\
\hline \multirow{2}{*}{ Outil } & Profil (âge, ancienneté, formation initiale...) \\
\cline { 2 - 2 } & Motivation et sentiment d'efficacité personnelle \\
\cline { 2 - 2 } & Pratiques habituelles et conceptions initiales \\
\cline { 2 - 2 } & Aspect matériel \\
\cline { 2 - 2 } & $\begin{array}{l}\text { Méthodologie (entrée par le guide méthodologique, structuration autorisant des choix) } \\
\text { principes...) }\end{array}$ \\
\hline \multirow{2}{*}{$\begin{array}{l}\text { Environnement didactique (structure, synthèses, consignes, ancrage dans les programmes, } \\
\text { orgationnel }\end{array}$} & Soutien de la direction \\
\cline { 2 - 2 } Classe & Collaboration \\
\cline { 2 - 2 } & Motivation et réaction face aux activités \\
\cline { 2 - 2 } $\begin{array}{l}\text { Soutien } \\
\text { externe } \\
\text { (recherche) }\end{array}$ & Climat de classe \\
\cline { 2 - 2 } & Formation \\
\hline
\end{tabular}

